\title{
Review of: "Emotions explain differences in the diffusion of true vs. false social media rumors"
}

nenggang xie

Potential competing interests: The author(s) declared that no potential competing interests exist.

This is study that examins the relationship between emotion (sentiment) and the spread of true and false rumors (information) on social media. It defined two categories of sentiment based on the big data: 1) positive and negative extreme sentiment and 2) anger, anticipation, disgust, fear, joy, trust, sadness and surprise. Then, the temporal state of these emotions in the propagation of true and false rumors (information) was analyzed by mathematical statistics. Some significant results were obtained from this study, and the established mathematical models were worthy of reference by management scientists, psychologists and sociologists:

There are a number of concerns with this study which include the following:

1. The data contained $\mathrm{N}=126,301$ rumor cascades on Twitter in 2006 through 2017 . The authors have screened and preprocessed the data (true vs. false rumor cascades). The dataset was huge and contained news from all sides, which is representative to a certain extent. The authors have made the dataset public, which provides valuable material for subsequent researchers.

2.Scores for sentiment and emotion were computed, and a regression model was constructed to analyze the cascade phenomenon of two extreme sentiment, eight emotions and true and false rumors. Furthermore, some interesting conclusions were obtained: for example, positive sentiment seems to promote the spread of false rumors, while negative sentiment promotes the spread of true rumors. Compared with true rumors, false rumors were mixed with more negative emotions, such as fear, disgust and surprise. The cascades of false rumors with words related to anticipation, anger, and trust are more widespread than true rumors. These meaningful conclusions are also consistent with the psychological state of rumor propagation in our life.

3.After the data results were obtained, two user studies were conducted to verify whether the data were consistent. Kendall's W approach was used to prove that the participants exhibited a statistically significant interrater agreement. Finally, the robustness was verified by fine-grained emotion checks and additional checks. Furthermore, they found that in all cases, the results were robust to support data reliability.

4. This study presented modeling and analysis methods of the relationship between emotions and rumor mongering. It is worthy of imitation and reference by other peer researchers. For example, the data are obtained by comparing the diffusion based on the complementary cumulative distribution functions (CCDFs). Kolmogorov-Smirnov (KS) tests confirmed that these data differences were statistically 
significant, in line with mathematical statistics methods, and post-processing renderings, etc. 